\title{
Grafito isotrópico obtenido a partir de coque para aplicaciones en automoción
}

\author{
J. OÑORO', F.J. MIELGO', C. RANNINGER' ${ }^{1}$, J. CASANOVA ${ }^{2}$ \\ 'Dept. Ingeniería y Ciencia de los Materiales. ETSI Industriales. U. Politécnica de Madrid. \\ ${ }^{2}$ Dept. Ingeniería Energética y Fluidomecánica. ETSI Industriales. U. Politécnica de Madrid.
}

\begin{abstract}
El grafito es una forma alotrópica del carbono, consistente en capas de átomos de carbono cristalizados en anillos hexagonales planos. Las partículas de coque utilizadas para sintetizar el grafito convencional son largas y estrechas, lo que da lugar a una estructura orientada de alta anisotropía tras un proceso de compactación o extrusión. Con una adecuada selección de materias primas y procesos de conformado es posible reducir dicha anisotropía, hasta eliminarla en la obtención del grafito isotrópico. La empresa Repsol-YPF, S.A., ha desarrollado un proceso para la fabricación de una mesofase poliaromática extraída por carbonización de residuos del refino del petróleo, a partir de la cual se obtiene un grafito isotrópico de altas prestaciones y bajo costo. Este grafito presenta propiedades térmicas, mecánicas, químicas y tribológicas muy superiores al grafito convencional; habiéndose propuesto su utilización para la fabricación de pistones, válvulas, cierres de bombas de agua y otros componentes en motores de automoción. Se han desarrollado pistones diseñados con este grafito isotrópico para un motor de gasolina. Estos pistones prototipo han sido fabricados y ensayados en motores a plena potencia y plena carga. En este trabajo se analizan las propiedades físicas, mecánicas y el comportamiento a fractura de este grafito isotrópico.
\end{abstract}

Palabras clave: grafito isotrópico, pistones, fractura.

\section{Isotropic graphyte produced from coke to automotive aplications}

The graphite is an allotropic form of the element carbon consisting of layers of hexagonally arranged carbon atoms in a planar condensed ring system. Coke particles used for conventional synthetic graphite products are long and narrow. These particles produce a preferred structural orientation in the product after the conventional uniaxial pressing or extrusion with high anisotropy. With a properly election of raw materials and process is possible reduce the anisotropy to obtain isotropic graphite. Repsol-YPF has produced a binderless polyaromatic mesophase from petroleum pitch, which has shown to be very appropriated as raw material for production of high strength isotropic graphite with high performance and low cost. This graphite have offered thermal, mechanical, chemical and wear properties higher than conventional graphite. The isotropic graphite obtained is suitable for making pistons, valves, turbojoints and other automotives components. The pistons design with this isotropic graphite has been developed for an automotive petrol engine. Piston prototypes were manufactured and tested in engines at full power and at full torque. This paper analysed the physical, mechanical and fracture properties of the isotropic graphite obtained.

Key words: isotropic graphite, pistons, fracture.

\section{INTRODUCCIÓN}

El grafito es una forma alotrópica del carbono, que cristaliza en el sistema hexagonal, formando capas de anillos hexagonales planos. Las uniones entre las capas son enlaces débiles por fuerzas de Van der Waals, con una distancia interatómica de $2,46 \AA$, frente a los enlaces fuertes dentro del anillo con una distancia de 1,42 ̊. El grafito artificial se fabrica generalmente a partir de coque de petróleo y otros residuos de carbono. Su estructura y propiedades dependen de su génesis y el proceso de fabricación $(1,2)$. Las partículas de coque utilizadas para sintetizar el grafito convencional son largas y estrechas, lo que da lugar a una estructura orientada de alta anisotropía tras un proceso de compactación o extrusión. La anisotropía de la celda unitaria se traslada a la estructura del grafito, pero no con la misma intensidad. Con una selección de las materias primas y los procesos de conformado se puede conseguir un amplio intervalo de anisotropía, o incluso eliminarla en el grafito isotrópico(1,3). Este grafito tiene una mayor densidad e isotropía que el grafito convencional, lo que le hace alcanzar unas mejores propiedades mecánicas, térmicas y de resistencia al desgaste, que permite utilizarlo en aplicaciones donde antes no era posible emplear el grafito convencional.

Los materiales utilizados en la fabricación de pistones y otros componentes en motores de combustión interna para automoción, deben soportar unas condiciones severas de servicio continuado. Dentro de cada cilindro se produce la combustión y por tanto se generan altas presiones y elevadas temperaturas durante dicha reacción. El material de los pistones debe soportar explosiones sucesivas y por tanto ciclos variables de carga durante todo el tiempo de funcionamiento del motor. En el arranque del motor se pasa de la temperatura ambiente a la temperatura de combustión en un tiempo muy corto. Dentro de cada pistón también se producen importantes gradientes térmicos. El grafito presenta notables ventajas frente a las aleaciones de aluminio utilizadas en la fabricación de pistones(4), por ser un material más ligero, tener un menor coeficiente de dilatación lineal y resistir temperaturas más elevadas. Sin embargo, el grafito convencional presenta una baja resistencia mecánica y elevada fragilidad que le hacen incapaz de cumplir los requerimientos exigidos a los pistones de automoción. El desarrollo del grafito isotrópico de mejores propiedades térmicas, mecánicas y tribológicas ha sido estudiado como una nueva alternativa tecnológica a los pistones de aluminio(4,5), aunque los costes actuales de fabricación le hacen inviable económicamente para esta aplicación.

En este trabajo se analizan las propiedades físicas, mecánicas y el comportamiento a fractura del grafito isotrópico obtenido y se comparan sus propiedades con el grafito convencional. También se analizan las primeras pruebas realizadas con pistones fabricados en este material funcionando en bancada.

\section{MATERIALES Y MÉTODOS.}

La empresa Repsol-YPF S.A., ha desarrollado un proceso para la fabricación de una mesofase poliaromática obtenida por carbonización de residuos del refino del petróleo. La obtención de un coque 
especial de alto contenido en compuestos volátiles, ha permitido la grafitización por Schunk, de un grafito isotrópico de alta densidad con elevadas propiedades mecánicas, que ha presentado una gran homogeneidad y bajo costo.

El grafito obtenido presenta propiedades térmicas, mecánicas, químicas y tribológicas muy superiores a las del grafito convencional, habiéndose propuesto su utilización para la fabricación de pistones, válvulas, cierres de bombas de agua y otros componentes en motores de automoción. La mejora de propiedades se debe al aumento de la densidad conjuntamente con una porosidad formada por cavidades de geometría esferoidal no interconectadas (Fig. 1), contrariamente a lo que ocurre en el grafito convencional, donde la conexión de los defectos da lugar a un material de elevada fragilidad.La densidad del grafito se ha determinado por medición y pesada, según la norma ASTM 559. La porosidad mediante análisis de imágen de probetas pulidas. El modulo de elasticidad se ha obtenido a partir de la velocidad de propagación de las ondas longitudinales ultrasónicas en el material. Los ensayos de resistencia a la flexión en tres puntos, de compresión y de tenacidad de fractura, se han realizado en una máquina universal de ensayos MTS. La tenacidad a la fractura se ha obtenido ensayando a flexión en tres


Estos ensayos fueron realizados según la norma provisional ASTM PS 70-97 para la determinación de la tenacidad a la fractura de materiales cerámicos avanzados a temperatura ambiente. En estos ensayos se determino el parámetro $\mathbf{K}_{\mathrm{Ipb}}$ como valor de la tenacidad a la fractura:

$$
\mathrm{K}_{\mathrm{Ipb}}=\mathrm{g} \cdot\left[\frac{\mathrm{P}_{\max } \cdot \mathrm{S}_{0} \cdot 10^{-6}}{\mathrm{~B} \cdot \mathrm{W}^{3 / 2}}\right] \cdot\left[\frac{3(\mathrm{a} / \mathrm{W})^{1 / 2}}{2(1-\mathrm{a} / \mathrm{W})^{3 / 2}}\right]
$$

donde $\mathbf{P}_{\max }$ es la carga máxima alcanzada, $\mathbf{S}_{0}$ la distancia entre apoyos, $\mathbf{W}$ el espesor de la probeta, $\mathbf{B}$ la anchura de la probeta, $\mathbf{g}=\mathbf{g}(\mathbf{a} / \mathbf{W})$ y a es la longitud de la pregrieta.

\section{RESULTADOS Y DISCUSIÓN}

En la Tabla I se recogen las propiedades físicas y mecánicas del nuevo grafito isotrópico obtenido comparado con el grafito convencional. En dicha tabla podemos ver la importante mejora de propiedades obtenidas. Todas las propiedades mecánicas han aumentado, en especial la tenacidad a la fractura, que ha experimentado un incremento a más del doble, con respecto al grafito convencional, y aunque sigue siendo un valor muy bajo, la mejora es muy significativa.

En especial cabe destacar la mejora de la compacidad, consistente en un aumento de la densidad con una porosidad que se reduce prácticamente a la mitad, lo que ha conducido a una significativa mejora de las propiedades mecánicas. Es sabido que la disminución de poro-

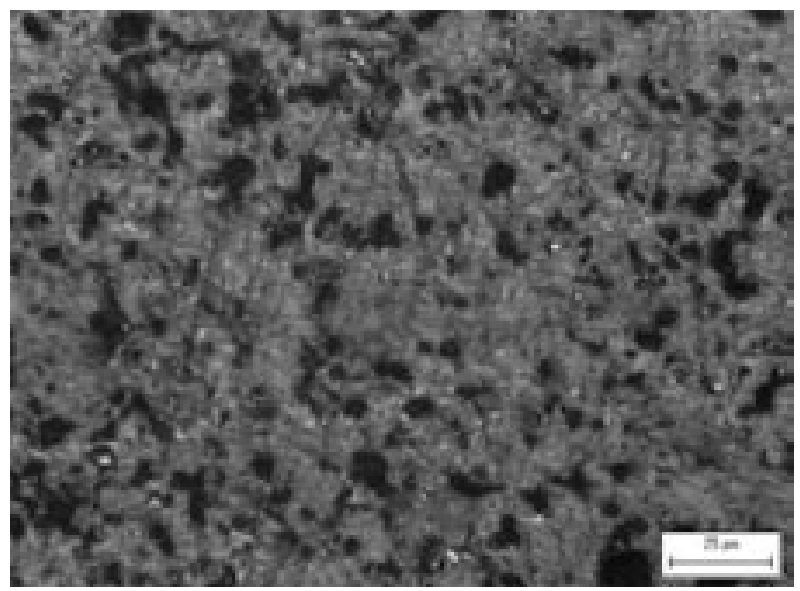

Figura 1: Microestructura del grafito isotrópico.
TABLA I.- PROPIEDADES DEL GRAFITO ISOTRÓPICO FRENTE AL CONVENCIONAL.

\begin{tabular}{|l|c|c|c|c|c|c|}
\hline Grafito & $\begin{array}{c}\text { Módulo } \\
\text { Elástico } \\
\mathrm{GPa}\end{array}$ & $\begin{array}{c}\text { Densidad } \\
\mathrm{g} / \mathrm{cm}^{3}\end{array}$ & $\begin{array}{c}\text { Porosidad } \\
\%\end{array}$ & $\begin{array}{c}\text { Resistencia } \\
\text { Compresión } \\
\mathrm{MPa}\end{array}$ & $\begin{array}{c}\text { Resistencia } \\
\text { Flexión } \\
\mathrm{MPa}\end{array}$ & $\begin{array}{c}\text { Tenacidad } \\
\text { de Fractura } \\
\mathrm{MPa} \mathrm{m}^{1 / 2}\end{array}$ \\
\hline Convencional & 17,1 & 1,79 & 21 & 59 & 81 & 0,88 \\
\hline Isotrópico & 20,5 & 1,95 & 11,6 & 66 & 97 & 2,05 \\
\hline
\end{tabular}

sidad en el grafito convencional produce una mejora de propiedades mecánicas(1,6); en este caso además de una reducción de la porosidad, se ha modificado la morfología de los poros obtenidos, más redondeados y de menores dimensiones, lo que ha contribuido también a la mejora de propiedades.

El grafito isotrópico obtenido ha alcanzado las propiedades físicas y mecánicas suficientes para ser utilizado para fabricar pistones. El Departamento de Ingeniería Energética y Fluidomecánica de la UPM ha rediseñado con grafito isotrópico a partir de pistones Renault, un nuevo pistón para motores de gasolina, con las propiedades de este nuevo material. Estos pistones han sido ensayados en la UPM, montados en un motor Renault adaptado con un único cilindro activo, unido a un motor eléctrico con variación de velocidad electrónica. Así mismo, otros pistones han sido montados por Renault en motores de gasolina en bancada, y han funcionado satisfactoriamente a plena carga durante 800 horas de forma ininterrumpida.

Aunque las pruebas de funcionamiento han sido muy prometedoras, durante el desarrollo del proyecto, se ha visto que la fabricación y utilización de pistones en serie, requiere implementaciones en función de una serie de problemas detectados. En primer lugar, sería necesario reducir los costes de mecanizado hasta hacerlos, al menos, semejantes a los de los pistones de aluminio. En segundo lugar, se han detectado desgastes acusados en zonas de máximo rozamiento y elevada temperatura, por lo que habría que modificar el diseño o incrementar la resistencia al desgaste del material. Y en tercer lugar, persiste una elevada fragilidad del grafito isotrópico, en comparación con el aluminio, lo que dificulta todas las operaciones de manipulación de los pistones.

\section{CONCLUSIONES}

El grafito isotrópico, obtenido a partir de efluentes de refinería, ha alcanzado unas propiedades físicas y mecánicas muy superiores a las obtenidas en el grafito convencional. Las principales mejoras han sido la disminución de la porosidad y el aumento de la tenacidad a la fractura.

El mejor comportamiento del grafito isotrópico obtenido ha permitido el diseño y la fabricación de pistones, así como su montaje y utilización a plena carga en motores de gasolina. Sin embargo, persiste una elevada fragilidad del grafito en comparación con las aleaciones de aluminio utilizadas en la fabricación de pistones.

\section{AGRADECIMIENTOS}

A la Comisión Europea por su financiación al proyecto MIGAC (BE97-4354).

\section{BIBLIOGRAFÍA}

1. P. Dollin, D.N. Ting and B. Rand, "Sintered carbons - A review", Extended Abstracts Carbon 96, 1996, 421.

2. E. Criado, M.F. Barba y J.M. Cardin. “Caracterización de grafitos empleados en refractarios", Bol.Soc.Esp.Ceram.V. 28 (1989) 2, 105-115.

3. A. Oya in: H. Marsh, E. A. Heintz, F. Rodríguez-Reinoso, Editors, "Introduction to carbon technologies", Ed. Universidad de Alicante. España, 561-578. 1997.

4. J. Haag, J. Heuer, M. Krämer, S. Pischinger, K. Wunderlich, J. Arndt, M. Stock and W. Coeling "Reduction of hydrocarbon emissions from SI-engines by use of carbon pistons" Coeling "Reduction of hy

5. S. Ashley, "Engine makers tap carbon-carbon pistons", Mechanical Engineering, May 5. S. As 1994 .

6. I.M. Pickup, B. Mc Enaney and R.G. Cooke. "Fracture processes in graphite and the effects of oxidation", Carbon, 24 [5] 535-543 (1986). 